Cite this: Phys. Chem. Chem. Phys., 2012, 14, 15078-15087

www.rsc.org/pccp

PAPER

\title{
Ultracold fluorine production via Doppler cooled BeF
}

\author{
Ian C. Lane* \\ Received 3rd August 2012, Accepted 10th September 2012 \\ DOI: $10.1039 / \mathrm{c} 2 \mathrm{cp} 42709 \mathrm{e}$
}

Large parts of the periodic table cannot be cooled by current laser-based methods. We investigate whether zero energy fragmentation of laser cooled fluorides is a potential source of ultracold fluorine atoms. We report new ab initio calculations on the lowest electronic states of the $\mathrm{BeF}$ diatomic molecule including spin-orbit coupling, the calculated minima for the valence electronic states being within $1 \mathrm{pm}$ of the spectroscopic values. A four colour cooling scheme based on the $\mathrm{A}^{2} \Pi \leftarrow \mathrm{X}^{2} \Sigma^{+}$transition is shown to be feasible for this molecule. Multi-Reference Configuration Interaction (MRCI) potentials of the lowest energy Rydberg states are reported for the first time and found to be in good agreement with experimental data. A series of multi-pulse excitation schemes from a single rovibrational level of the cooled molecule are proposed to produce cold fluorine atoms.

\section{Introduction}

Ultracold molecules and atoms offer a unique opportunity to study chemical reactions at the quantum state level. Attractions include the ability to select individual rovibrational states of the reagents, the elimination of translational energy spread and the control of product quantum states using external fields. However, there has only been one reactive collision studied in this way. ${ }^{1}$ Problems with exploiting these reagents include the difficulty in cooling many of the elemental groups of the periodic table. For example, reactions involving halogen atoms are very rapid ${ }^{2}$ but Doppler cooling such atoms would require continuous-wave lasers operating in the VUV or XUV at milliWatt powers. Another practical problem concerns the very reactivity of many p-block elements that naturally makes them of special interest to chemists. This aggressive behaviour makes them unsuitable for the high vacuum apparatus used in today's cold atom experiments. ${ }^{3}$ In particular, fluorine is known to ruin the non-evaporated getter (NEG) surfaces of modern UHV pumps. What is required is a method to transport these reactive species to the centre of a trap without destroying the chamber, whereupon they can be released for study. Delivering the halogens in the form of a trapped, ultracold, parent molecular gas prior to in situ laser fragmentation elegantly resolves both these problems.

Trapped ultracold fluorine atoms could be used as a source of highly reactive atoms for nanolithography. Wright and Lane $^{4}$ have proposed studying the reaction of vibrationally excited ultracold $\mathrm{Li}_{2}$ dimers with fluorine atoms and have suggested quantum state selection of the reaction products is

School of Chemistry and Chemical Engineering,

Queen's University Belfast, Stranmillis Road, Belfast, UK BT9 5 AG.

E-mail: i.lane@qub.ac.uk possible by selecting the initial vibrational state. Unfortunately, the production of trapped, cold halogen atoms presents perhaps one of the stiffest challenges for ultracold science. In particular, fluorine atoms are impossible to laser cool with current techniques as the lowest electric-dipole spin-allowed transition $3 \mathrm{~s}^{2} \mathrm{P}_{3 / 2} \leftarrow 2 \mathrm{p}^{4} \mathrm{P}_{3 / 2}\left(104731 \mathrm{~cm}^{-1}\right)$ lies below $96 \mathrm{~nm}$, a prohibitively short wavelength for a continuous wave laser. Furthermore, a single photon at this energy would impart a considerable momentum kick during spontaneous emission, leading to a significant recoil temperature. Hopes of using excited fluorine atoms and the $3 \mathrm{p}^{4} \mathrm{D}_{7 / 2}{ }^{0} \leftarrow 3 \mathrm{~s}^{4} \mathrm{P}_{5 / 2}$ transition at $685 \mathrm{~nm}$ were dashed ${ }^{6}$ by the relatively short lifetime $(3.7 \mu \mathrm{s})$ of the metastable $3 \mathrm{~s}^{4} \mathrm{P}_{5 / 2}$ state. Frustratingly, this lifetime is also too long for efficient optical cycling from the ground state. However, following the recent demonstration by Shuman et al. of Doppler cooled diatomic $\mathrm{SrF}^{7}$ it will not be long before trapped, ultracold fluorides can be prepared in the laboratory. Zero-energy fragmentation of such diatomics is a potential source of ultracold and trapped fluorine atoms.

The alkaline earth diatomics are ionic compounds or, more precisely, they have an ion-pair potential as the lowest electronic state. SrF has a Franck-Condon (FC) factor between the lowest vibrational levels of the $\mathrm{A}^{2} \Pi \leftarrow \mathrm{X}^{2} \Sigma^{+}$transition $^{8}$ of $f_{00}>0.98$ making this a highly diagonal transition. Nevertheless, three vibronic transitions need to be pumped in order to close the cooling cycle. Ab initio work by Ornellas ${ }^{9}$ calculated the corresponding FC factors for $\mathrm{BeF}$ but revealed that the $f_{00}$ value is approximately 0.90 , much lower than $\mathrm{SrF}$ and consequently superficially appears much less suitable for cooling. On the contrary, we demonstrate that there is a viable laser cooling scheme based on four vibronic transitions similar to the one successfully deployed for $\mathrm{SrF}$.

Unlike Doppler cooling of simple atoms, direct laser cooling of a molecular gas is complicated by its initial temperature, 
not because of the Maxwell Boltzmann velocities but the Boltzmann distribution of internal states. The larger energy separations between rovibrational levels in $\mathrm{BeF}$ will maximise the population in low $v$ and $J$ states when compared with heavier analogs like $\mathrm{SrF}$ or $\mathrm{BaF}$. From a kinematic perspective, $\mathrm{BeF}$ is also the most attractive parent for the production of ultracold fluorine because its partner fragment has a smaller mass, and therefore will extract the majority of any surplus centre-of-mass dissociation energy. However, a viable transition pathway must be found to link the lowest vibrational levels of the ground state with the long range vibrational states at the dissociation limit. Such states form the basis of the Feshbach resonances required to access the continuum scattering states corresponding to the free atoms. Realistically, this population transfer must be done through an excited electronic state (to effect a great change in the internuclear separation) and key requirements are good FC factors and practical excitation energies for the efficient transfer of internal population. In this paper we use $a b$ initio quantum chemistry to develop full potential curves of the lowest electronic states in $\mathrm{BeF}$ and we identify a viable fragmentation scheme to produce zero-energy fluorine atoms suitable for trapping.

Most of the detailed spectroscopic information of this fluoride is relatively dated and cannot match the quality of modern high resolution measurements. Datta ${ }^{10}$ first reported its spectra in 1922, identifying a bandhead at $309 \mathrm{~nm}$ with further bands extending to shorter wavelengths. Further analysis by Jenkins ${ }^{11}$ proved the emission was ${ }^{2} \Pi \rightarrow{ }^{2} \Sigma^{+}$and measured the excited state bond length as $1.39 \AA$. The author assigned the spin-orbit $\mathrm{A}^{2} \Pi_{1 / 2}$ state as lowest in energy but a later analysis of $\Lambda$-coupling constants by Walker and Barrow ${ }^{12}$ suggested that it is the $\mathrm{A}^{2} \Pi_{3 / 2}$ component that is lower. This issue was finally resolved by theoretical analysis ${ }^{13}$ based on $a b$ initio quantum chemistry. In 1965 Rao et al. reported ${ }^{14}$ emission spectra corresponding to the $\mathrm{C}^{2} \Sigma^{+} \rightarrow \mathrm{A}^{2} \Pi$ transition while a VUV study of the $\mathrm{B}^{2} \Sigma^{+}$and $\mathrm{C}^{2} \Sigma^{+}$electronic states was published two years later by Novikov and Gurvich. ${ }^{15}$ The spectroscopy of the $\mathrm{B}^{2} \Sigma^{+}$state was studied in further detail by Tai and Verma ${ }^{16}$ but only the lowest three vibrational levels have been interrogated in any great detail. The Russian study also reported red degraded bands at $64000 \mathrm{~cm}^{-1}$ and $66000 \mathrm{~cm}^{-1}$ attributed to a pair of excited ${ }^{2} \Pi$ states but Tai and Verma indicated they did not observe these bands in their experiments.

Quantum chemistry is used here to address a number of key issues regarding the proposed method to form cold atoms, such as the availability of a barrier-less potential suitable for zero-energy fragmentation and whether the highest vibrational levels of this potential can be efficiently populated via accessible optical transitions. To answer these questions accurate potentials are naturally required but an additional motivation for this study is to provide generic valence potentials useful for the interpretation of the other alkaline fluorides too. The alkaline earth metals are not well served by the current generation of basis sets (even the best Be set is relatively small compared to other second row elements) and thus the lightest molecule in the series is the logical choice for reliable potentials.

Previous theoretical work has naturally concentrated on the lowest pair of electronic states. In particular, there has been some debate on the strength of the chemical bond and on the nature of the spin-orbit coupling in the $\mathrm{A}^{2} \Pi$ state. Roach and Kuntz calculated a dissociation energy $D_{\mathrm{e}}$ of $3.94 \mathrm{eV}^{17}$ for the $\mathrm{X}^{2} \Sigma^{+}$state which differed considerably from the experimental values of Hildenbrand and Murad ${ }^{18}(5.85 \mathrm{eV})$ and Farber and Srivastava $^{19}(6.26 \mathrm{eV})$. A recent $\operatorname{CCSD}(\mathrm{T})$ study by Dixon is currently the most reliable theoretical study ${ }^{20}$ and reports the dissociation energy as $5.87 \mathrm{eV}$. Walker and Richards ${ }^{21}$ demonstrated that the observed spin-orbit coupling is consistent ${ }^{22}$ with a predominantly $\pi^{4} \pi$ configuration in the $\mathrm{A}^{2} \Pi$ state with a considerable $\pi^{3} \sigma^{2}$ contribution and later work by Cooper, Prosser and Richards ${ }^{13}$ reconciled this picture with the $\Lambda$-coupling constants observed by Walker and Barrow. The spin-orbit coupling was later addressed at the MRCI level by Marian ${ }^{23}$ who also calculated constants for the $\mathrm{D}^{2} \Pi$ state. The MRCI method also formed the basis of later studies on the dissociation energy $^{24}$ and dipole moment ${ }^{25}$ of the $\mathrm{X}^{2} \Sigma^{+}$state. Such ab initio potentials were used by Ornellas et al. to calculate the ground ${ }^{26}$ and $\mathrm{A}^{2} \Pi$ vibronic levels, rotational constants and lifetimes. ${ }^{27}$ The calculated FC factors suggest that the fundamental $0-0$ transition has a much lower value than the equivalent vibronic transition in SrF. These calculations were repeated with better basis sets by Ornellas in 2005, confirming this result, while a V5Z/AV6Z study by Zhu et al. has recently been published. ${ }^{28}$ This final study also included the vibrational levels and spectroscopic constants of the $\mathrm{B}^{2} \Sigma^{+}$state but did not address transition dipoles or higher excited states. Previous theoretical studies have, in general, paid little attention to the excited state asymptote $\mathrm{Be}\left({ }^{3} \mathrm{P}\right)+\mathrm{F}\left({ }^{2} \mathrm{P}\right)$. The only published potentials that illustrate this asymptote are those from Prosser and Richards, ${ }^{29}$ which are only computed at the Hartree-Fock level. Populating a vibronic level close to this dissociation limit may be crucial to the production of cold fluorine and it is therefore necessary to identify if a suitable bound state is available.

\section{Ab initio calculations}

The electronic states are found using Complete Active Space SCF (CASSCF) wavefunctions ${ }^{30}$ based on the valence orbital space $6 a_{1}, 2 b_{1}$ and $2 b_{2}(6220)$, though the lowest two $a_{1}$ orbitals are treated as the core throughout. Dynamic electron correlation was calculated by Multi-Reference Configuration Interaction ${ }^{31}$

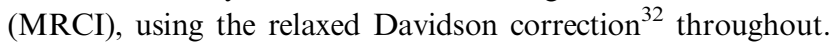
These calculations were augmented with additional points using the larger active spaces 7320 (for the Rydberg component of the $\Pi$ states) and 8220 (for the $\Sigma^{+}$states). Only states of doublet multiplicity were calculated, and since all the ${ }^{2} \Sigma^{-}$valence states are repulsive they are not discussed further. Currently, the largest available correlation consistent basis $\operatorname{set}^{33}$ for $\mathrm{Be}$ is cc-pV5Z (V5Z) which lacks the diffuse functions for an accurate description of excited atomic levels of Be. The augmented cc-pV6Z (AV6Z) basis set is used for $F$ and all calculations were performed using the MOLPRO suite of programs. ${ }^{34}$

\section{Results}

(a) $\mathbf{A}^{2} \Pi \leftarrow \mathrm{X}^{2} \Sigma^{+}$transition and laser cooling cycle

The $a b$ initio potentials obtained are shown in Fig. 1. The ab initio data points were fitted using the LEVEL program (version 8.0) ${ }^{35}$ 

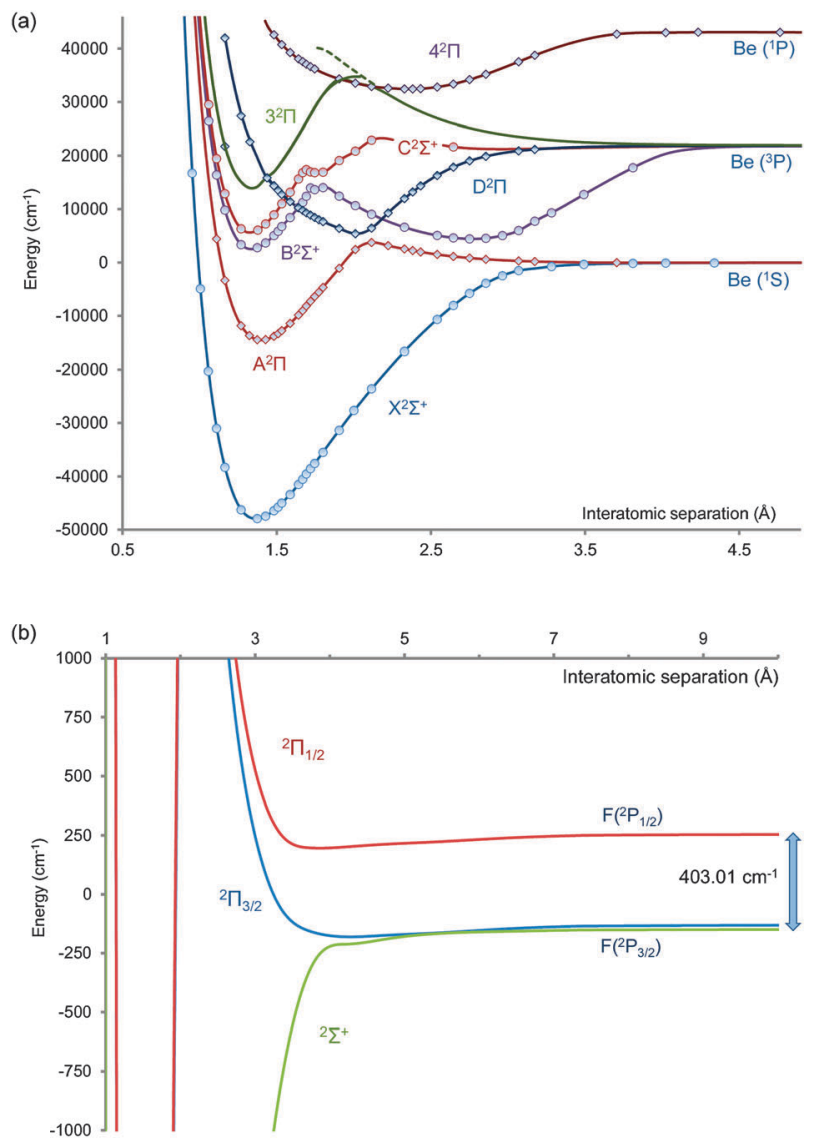

Fig. 1 Valence MRCI potential energy curves of BeF calculated with V5Z basis set on Be and AV6Z basis set on F. Circles are ab initio points for ${ }^{2} \Sigma^{+}$states and diamonds for ${ }^{2} \Pi$. Be $\left(2^{3} \mathrm{P}\right)$ dissociation limit calculated as $22070 \mathrm{~cm}^{-1}$ (experimental value $21980 \mathrm{~cm}^{-1}$ ) and $\operatorname{Be}\left({ }^{1} \mathrm{P}\right.$ ) as $43504 \mathrm{~cm}^{-1}$ (experimental value $42565 \mathrm{~cm}^{-1}$ ). (b) Spin-orbit potentials associated with the fluorine ground state. to obtain the bound rovibronic levels, spectroscopic constants (Table 1), FC factors and radiative lifetimes. The calculated $v=1-v=0$ energy separation in the $\mathrm{X}$ state is $1235.11 \mathrm{~cm}^{-1}$ in excellent agreement with the experimental value ${ }^{16}$ of $1246.70 \mathrm{~cm}^{-1}$. A similar relative error is found in the values of $T_{00}$, where the experimental value is $33187 \mathrm{~cm}^{-1}$ and the calculated one is $33511 \mathrm{~cm}^{-1}(0.9 \%$ discrepancy). The discrepancies between the calculated and experimental $r_{\mathrm{e}}$ values are $<0.01 \AA$. The calculated bond dissociation energy $\mathrm{D}_{0}\left(\mathrm{X}^{2} \Sigma^{+}\right)$is $47092 \mathrm{~cm}^{-1}$ $(5.838 \mathrm{eV})$ and $\mathrm{D}_{0}\left(\mathrm{~A}^{2} \Pi\right)$ is $13578 \mathrm{~cm}^{-1}(1.683 \mathrm{eV})$, the former within $1 \%$ of a recent high level $\operatorname{CCSD}(\mathrm{T})$ based calculation $\left(47350 \mathrm{~cm}^{-1}\right)$ and smaller than the value reported by Zhu et al. Consistent with this, we find that the highest computed vibrational level is $v=64$, within $1.5 \mathrm{~cm}^{-1}$ of the dissociation limit, rather lower than $v=74$ reported in that work. Experimental $\mathrm{X}^{2} \Sigma^{+}$data only exists for vibrational levels up to $v=15\left(17327 \mathrm{~cm}^{-1}\right)$.

Like Ornellas et al., we calculate as many as 16 vibrational levels in the $A^{2} \Pi$ state, though only 13 have been experimentally observed. We find that $v=13$ is the last $\mathrm{A}^{2} \Pi$ vibrational level below the $\operatorname{Be}\left({ }^{1} \mathrm{~S}\right)+\mathrm{F}\left({ }^{2} \mathrm{P}\right)$ asymptote. Unlike the earlier study, we also computed the spin-orbit coupling in the $\mathrm{A}^{2} \Pi$ state and used the lower component to determine the $\mathrm{FC}$ factors with the ground state. These are in good agreement with Ornellas (Fig. 2a) but despite the relatively small value of $f_{00}$, a four colour laser cooling scheme can be found thanks to the additional spontaneous emission channels being tightly clustered around the $0-0$ transition (Fig. 2b). The sum of all transitions beyond $v=3$ is $<6 \times 10^{-5}$, similar to SrF. Fig. 2a also demonstrates the relative strengths of transition with $\Delta v=0, \pm 1$ and \pm 2 , the latter clearly increasing at higher $v^{\prime \prime}$. The relative strength of the $\Delta v=0$ transitions falls to a minimum for $v^{\prime \prime}=8$ before rising again. The relative weakness of $\Delta v= \pm 3$ or higher explains why vibrational data on the

Table 1 A comparison between the calculated constants (V5Z/AV6Z basis set, this work) and experimental values (square bracketed values) for a variety of constants determined for the electronic states of the BeF radical. $T_{\mathrm{e}}$ is the energy of the potential minimum for the specified electronic state, $r_{\mathrm{e}}$ is the internuclear separation at the minimum, $T_{\mathrm{v}}$ is the energy of the vibrational level $v$ above the potential minimum (the calculated $T_{\mathrm{v}}$ values assume $J=0$ ) and $B_{\mathrm{v}}$ is the rotational constant for each vibrational level. Experimental data from T. E. H. Walker and R. F. Barrow, The $\mathrm{A}^{2} \Pi \leftarrow \mathrm{X}^{2} \Sigma^{+}$system of BeF, J. Phys. B, 1969, 2, 102 unless stated otherwise

\begin{tabular}{|c|c|c|c|c|c|}
\hline $\mathrm{BeF}$ & $T_{\mathrm{e}}\left(\mathrm{cm}^{-1}\right)$ & $r_{\mathrm{e}}(\AA)$ & $v$ & $T_{\mathrm{v}}\left(\mathrm{cm}^{-1}\right)$ & $B_{\mathrm{v}}\left(\mathrm{cm}^{-1}\right)$ \\
\hline$\overline{\mathrm{X}^{2} \Sigma^{+}}$ & $\overline{0}$ & $\begin{array}{l}1.364 \\
{[1.3609]} \\
{[1.3610]^{b}}\end{array}$ & $\begin{array}{l}0 \\
1 \\
2 \\
3\end{array}$ & $\begin{array}{l}617.15[630.5]^{c} \\
1852.26[1877.7]^{c} \\
3077.67[3106.7]^{c} \\
4276.50[4317.5]^{c}\end{array}$ & $\begin{array}{l}1.4658[1.4801] \\
1.4481[1.4625] \\
1.4312 \\
1.4157\end{array}$ \\
\hline $\mathrm{A}^{2} \Pi$ & $\begin{array}{l}33542.8 \\
{[33234]}\end{array}$ & $\begin{array}{l}1.399 \\
{[1.394]}\end{array}$ & $\begin{array}{l}0 \\
1 \\
2\end{array}$ & $\begin{array}{l}588.45[585.6] \\
1755.74[1740.3] \\
2907.62\end{array}$ & $\begin{array}{l}1.4043[1.4115] \\
1.3887[1.3939] \\
1.3706\end{array}$ \\
\hline $\mathrm{B}^{2} \Sigma^{+}$ & $\begin{array}{l}50182.8 \\
{[49503.2]^{a}}\end{array}$ & $\begin{array}{l}1.334 \\
{[1.328]^{a, d}}\end{array}$ & $\begin{array}{l}0 \\
1 \\
2^{e}\end{array}$ & $\begin{array}{l}680.88[777.63]^{a} \\
1999.35[2113.84]^{a} \\
3293.69[3423.77]^{a}\end{array}$ & $\begin{array}{l}1.5347[1.5531]^{a} \\
1.5090[1.5329]^{a} \\
1.4[1.5118]^{a}\end{array}$ \\
\hline $\mathrm{B}^{2} \Sigma^{+}$(outer) & 52117.8 & 2.775 & $\begin{array}{l}0 \\
1 \\
2\end{array}$ & $\begin{array}{l}201.8 \\
600.6 \\
993.2\end{array}$ & $\begin{array}{l}0.362 \\
0.368 \\
0.373\end{array}$ \\
\hline $\mathrm{D}^{2} \Pi$ & 53249 & 2.015 & $\begin{array}{l}0 \\
0 \\
1\end{array}$ & $\begin{array}{l}520.4 \\
1476.6\end{array}$ & $\begin{array}{l}0.6838 \\
0.7018\end{array}$ \\
\hline $\mathrm{C}^{2} \Sigma^{+}$ & $\begin{array}{l}53295 \\
{[50419]}\end{array}$ & $\begin{array}{l}1.324 \\
{[1.322]^{a}}\end{array}$ & $\begin{array}{l}0 \\
1\end{array}$ & $\begin{array}{l}736.4[652.5]^{a} \\
2173.6[2049.4]^{a}\end{array}$ & $\begin{array}{l}1.559[1.569]^{a} \\
1.534[1.551]^{a}\end{array}$ \\
\hline $3^{2} \Pi$ & 61612 & 1.340 & $\begin{array}{l}0 \\
1\end{array}$ & $\begin{array}{l}821.0 \\
2388.5\end{array}$ & $\begin{array}{l}1.541 \\
1.529\end{array}$ \\
\hline
\end{tabular}

${ }^{a}$ G. Tai and R. D. Verma, The UV Spectrum of BeF, J. Mol. Spec., 1995, 173, 1. ${ }^{b}$ M. M. Novikov and L. U. Gurvich, Optics Spectrosc., 1966, 23, 173. ${ }^{c}$ V. M. Tatevskii, L. N. Tunitskii and M. M. Novikov, Optika Spectrosk., 1958, 5, 520. ${ }^{d}$ From $r_{0}=1.335 .{ }^{e}$ Calculated as $v=6$ (see text). 
(a)

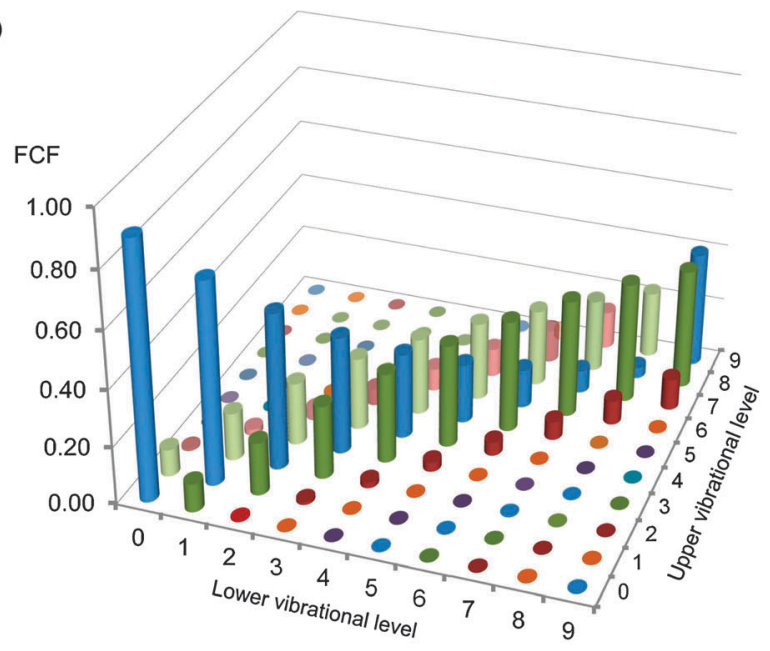

(b)

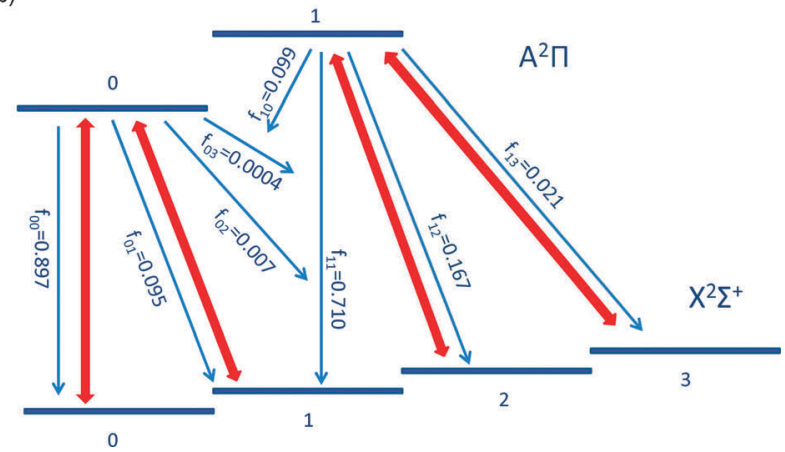

Fig. 2 (a) Calculated Franck-Condon factors (FC factors) in BeF for the lowest ten vibrational levels of the cooling transition $A^{2} \Pi \leftarrow X^{2} \Sigma^{+}$ transition. Diagonal transitions in blue, $\Delta v= \pm 1$ in green and $\Delta v= \pm 2$ in red. (b) Proposed laser cooling scheme for BeF using the $\mathrm{A}^{2} \Pi \leftarrow \mathrm{X}^{2} \Sigma^{+}$transition. For each vibronic transition, only one rotational transition needs to be excited. The decay pathways are shown as single headed arrows while the cooling frequencies are represented by double headed ones. The Franck-Condon factors (FC factors) are the ones calculated in the present study. Both $\mathrm{Be}(I=3 / 2)$ and $\mathrm{F}(I=1 / 2)$ have nuclear spin leading to hyperfine structure (not illustrated). For each upper vibrational level, the sums over the remaining FC factors are $f_{03}+<0.00006$ and $f_{13+}<0.0025$.

$\mathrm{X}^{2} \Sigma^{+}$state is limited to $v=15$ as the $\mathrm{A}^{2} \Pi \leftarrow \mathrm{X}^{2} \Sigma^{+}$transition is used in such studies. Large FC factors, however, are not enough to ensure a good cooling candidate as the rate of optical cycling must also be significant $\left(10^{5}-10^{8} \mathrm{~s}^{-1}\right)$ to produce a strong Doppler force. The line strength (Einstein A coefficient) for the primary cooling transition is $1.35 \times 10^{8} \mathrm{~s}^{-1}$, giving a lifetime of 7.9 ns (about $1 / 3$ that of the transition in $\mathrm{SrF}$ ).

The hyperfine structure of ${ }^{9} \mathrm{BeF}(100 \%$ abundance) is more complex than in ${ }^{88} \mathrm{SrF}(82.5 \%$ of a natural sample) because the former metal has a nuclear spin of $I=3 / 2$ (the same as ${ }^{135} \mathrm{Ba}$ and $\left.{ }^{137} \mathrm{Ba}^{36}\right)$ and this will complicate the laser cooling process. The rovibronic transitions in $\mathrm{BeF}$ can be labeled ${ }^{2} \Pi\left(\right.$ case $\left.a_{\alpha, J}\right) \leftarrow{ }^{2} \Sigma^{+}\left(\right.$case $\left.b_{\beta J}\right)$ and the Fermi contact term $b_{\mathrm{F}}$ in $\mathrm{BeF}^{37}$ has been reported as $\mathrm{b}_{\mathrm{F}}(\mathrm{Be})=0.0098 \mathrm{~cm}^{-1}$. There are 7 hyperfine levels associated with ${ }^{2} \Sigma^{+}(J=3 / 2) N=1$ and 4 with ${ }^{2} \Sigma^{+}(J=1 / 2) N=1$ and these levels need to be pumped to complete the cooling cycle. In the excited state, an additional complication is the splitting of each rotational level ( $\Lambda$-type doubling) as a consequence of the non-zero orbital electronic motion $\Lambda$. However, only one component of the $\Lambda$-doublet is populated during the cooling cycle (parity selection rule) and the hyperfine energy shifts can be compensated using acousto-optic modulators. In order to maximize the density of cooled molecules, a small internal population spread amongst the vibrational and rotational levels is desirable. Thus, perhaps a little paradoxically, it is the initial internal temperature that has a greater influence on the cooling of molecules than the translational temperature. Buffer gas cooling techniques can presently create intense beams $\left(10^{11} \mathrm{~cm}^{-3}\right.$ density) of even large molecules like naphthalene at temperatures of $6 \mathrm{~K}^{38}$

Laser cooling creates a translationally cold molecule and ergo (almost) zero energy fragmentation of such molecules in a trap will retain the initial thermal energy of the ultracold parents. Such a scheme has been proposed by Wells and Lane for the production of $\mathrm{C}$ atoms from laser cooled $\mathrm{CH}$ radicals. ${ }^{39}$ The long range hyperfine structure in $\mathrm{BeF}$ is determined by the splitting in the ground ${ }^{2} \mathrm{P}_{1 / 2}$ spin-orbit state of fluorine $(I=1 / 2, \Delta E=4.02 \mathrm{GHz})$ and its coupling with the nuclear spin on Be. The result is four hyperfine levels $(F=5 / 2,3 / 2,3 / 2$ and $1 / 2$ ) and their presence opens the door to populating a quasi-bound level above the lowest dissociation limit that can fragment into free atoms. It is necessary, therefore, to transfer the molecular population efficiently into this state from one of the vibrational levels populated via the laser cooling process (we chose $v^{\prime \prime}=2$ for this study). A crucial issue is the long range behaviour of the potentials, since the presence of a barrier along the dissociation path will lead to unavoidable heating of the gas as the excess energy required to climb the barrier will have to be released in the form of kinetic energy in the atomic fragments. Therefore, we computed the spin orbit coupling for the molecular potentials correlated to the ground asymptote, using a VQZ basis set on each atom. By including just the $\mathrm{X}^{2} \Sigma^{+}$and $\mathrm{A}^{2} \Pi$ states in the calculation we found poor agreement between the long range $(50 \AA)$ splitting and the known value for atomic $F\left(286 \mathrm{~cm}^{-1}\right.$ versus the experimental value of $404.141 \mathrm{~cm}^{-1}$ ), but by extending the calculation to include the $\mathrm{B}^{2} \Sigma^{+}$and $\mathrm{D}^{2} \Pi$ states as well, we calculated a value of $403.01 \mathrm{~cm}^{-1}$, in excellent agreement. ${ }^{40}$ The long range energies with spin-orbit coupling are shown in Fig. $1 \mathrm{~b}$ and reveal no evidence of a barrier on the $\mathrm{X}^{2} \Sigma^{+}$potential curve.

\section{(b) STIRAP population transfer via $\mathrm{B}^{2} \Sigma^{+}$state}

Stimulated Rapid Adiabatic Passage ${ }^{41}$ (STIRAP), a fully coherent optical procedure for population transfer, has been used previously for the formation of ultracold molecules ${ }^{42}$ from cold atoms. For that application, a molecular rovibrational level above the atomic asymptote must be brought into resonance with the kinetic energy of colliding laser cooled atoms (creating a Feshbach resonance). A counterintuitive sequence of optical pulses then transfers the population from the quasi-bound state to a deeply bound rovibrational level. The STIRAP method produces low loss rates as adiabatic passage suppresses any decay via the intermediate excited state as demonstrated by $\mathrm{Ye}^{43}$ and co-workers for RbK. By reversing the 
pulse sequence and so using STIRAP to transfer molecules between the bound state and asymptote, microscopic reversibility ensures that zero-energy (centre of mass frame) atomic products can be formed with high transfer efficiency from a single quantum state in an ultracold molecule. While STIRAP has not been applied directly in this way, experiments ${ }^{44}$ with $\mathrm{Cs}_{2}$ by Danzl et al. have clearly demonstrated efficient population transfer back into a Feshbach resonance. It is important to note that we are not proposing excitation into a continuum of scattering states, but into a quasi-bound rovibrational level, the coupling of which with the continuum of atoms can be adjusted by external fields. The initial single quantum level of the ultracold molecule naturally does not have to be formed itself by STIRAP association of atoms, and the proposed method involves initial state preparation by direct laser cooling of a molecule like BeF.

The states relevant for STIRAP production of fluorine atoms are presented in Fig. 3a, where the highest calculated
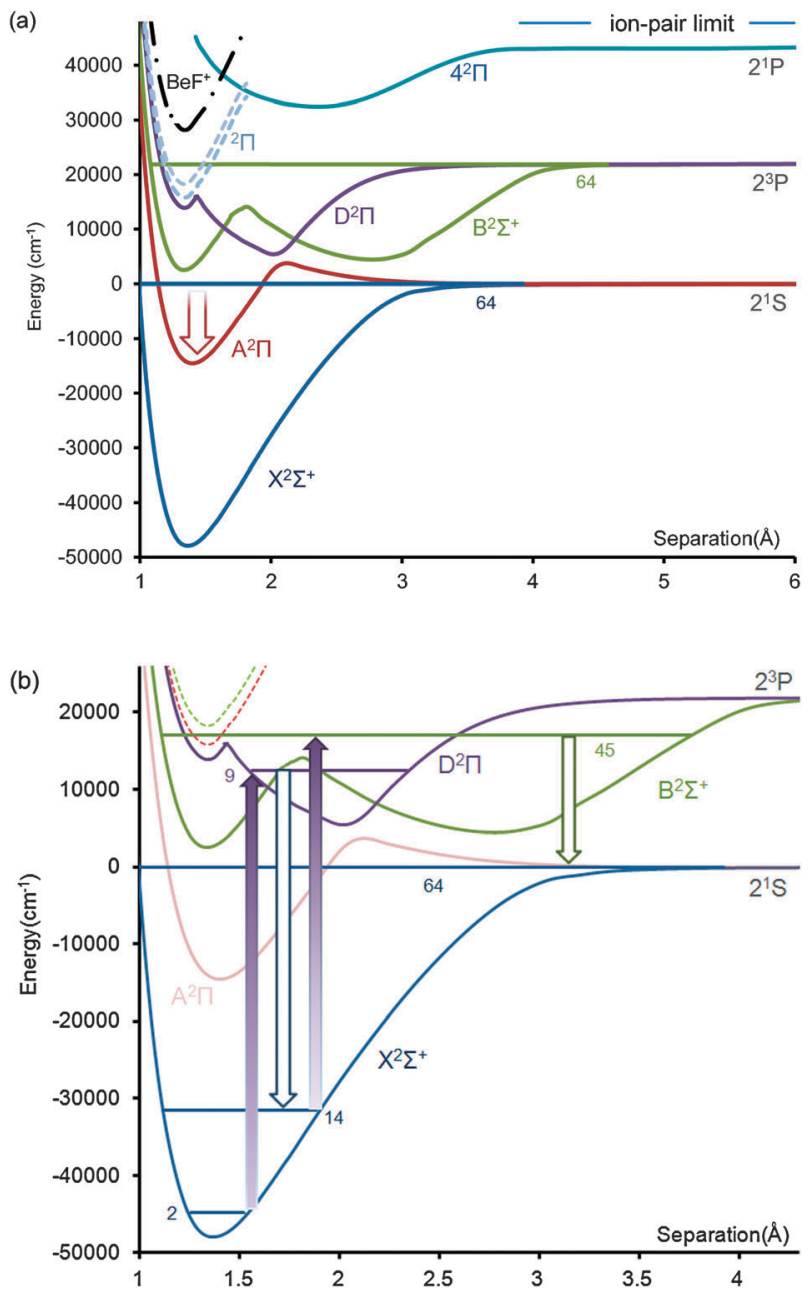

Fig. 3 STIRAP pathway to produce cold fluorine atoms via the $\mathrm{B}^{2} \Sigma^{+}$state. (a) The states relevant for this study. In addition to those calculated, schematic versions of the two observed Rydberg states observed by Gurvich are shown, and the ground state of the $\mathrm{BeF}^{+}$ion using the calculated dissociation energy reported by Ornellas et al. Also shown is the ion-pair asymptote $\mathrm{Be}^{+}\left({ }^{2} \mathrm{~S}\right)+\mathrm{F}^{-}\left({ }^{1} \mathrm{~S}\right)$; (b) Four pulse scheme through $\mathrm{D}^{2} \Pi$ and $\mathrm{B}^{2} \Sigma^{+}$states. The PUMP pulses are represented by the filled arrows. vibrational states are shown for the $\mathrm{X}^{2} \Sigma^{+}$and $\mathrm{B}^{2} \Sigma^{+}$states (in both cases this is, coincidently, $v=64$ ). In our study we use the calculated excited vibrational levels as surrogates for the Feshbach states (dominated by long-range dispersion forces) to explore possible excitation pathways and decay modes for the highly excited vibrational levels. For example, direct excitation to the highest vibrational level of the $\mathrm{B}^{2} \Sigma^{+}$state would appear to be a simple way for creating cold ground state fluorine atoms because although it is a higher energy asymptote it is the Be atom alone that is formed in an electronically excited state. Sadly, calculation of the spontaneous emission rate from $\mathrm{B}^{2} \Sigma^{+} v^{\prime}=64$ (Fig. 4a) reveals that the Feshbach will decay rapidly by photon emission rather than fragmentation into cold atoms. The corresponding Feshbach levels along the ground asymptote are the most suitable, and clearly only the $\mathrm{X}^{2} \Sigma^{+}$potential lacks a barrier. One complication is that since efficient laser cooling of molecules requires an excited state (here $\mathrm{A}^{2} \Pi$ ) below the lowest asymptote (to prevent predissociation destroying the cooling cycle), spontaneous emission from the threshold vibrational level back into this state is also unavoidable. However, we calculated the total
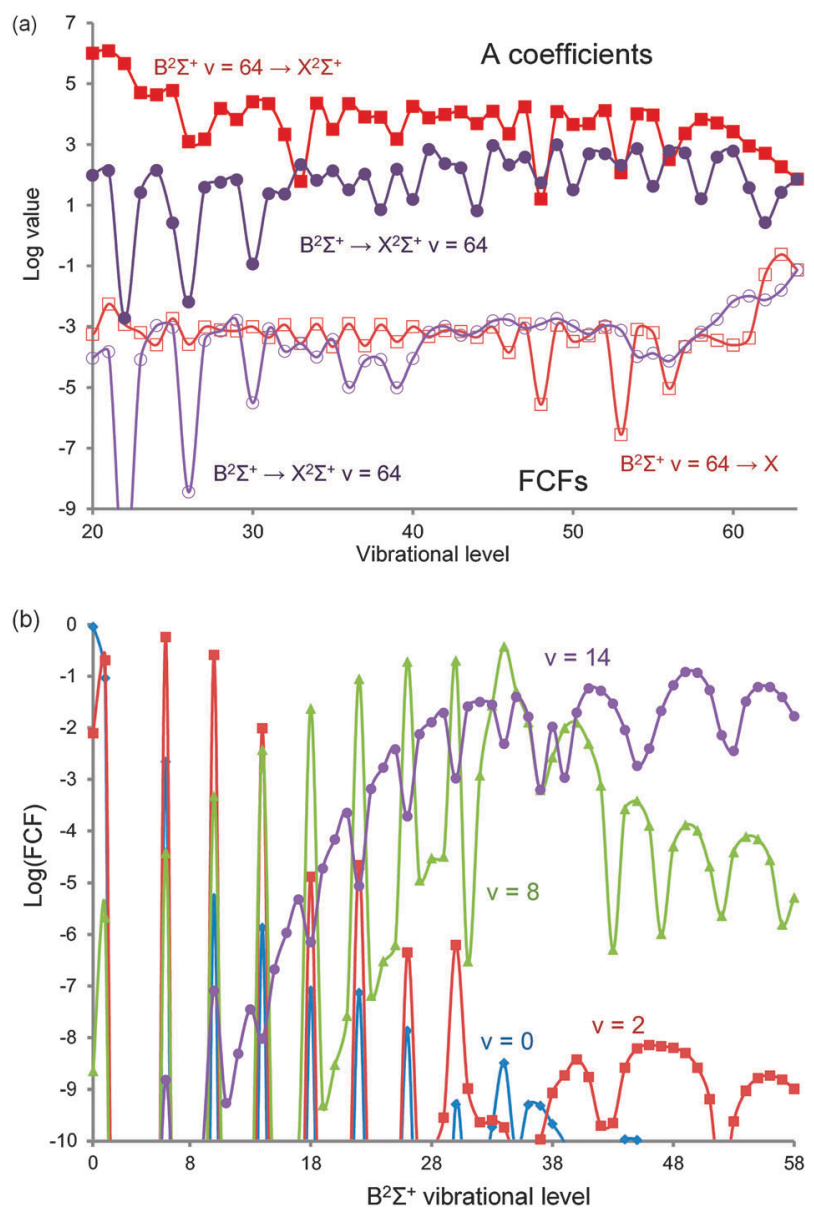

Fig. 4 Calculated Franck-Condon factors (FC factors) in BeF as a function of vibrational quantum number. (a) The FC factors and A coefficients for vibronic transitions involving the $v=64$ states of the $\mathrm{B}^{2} \Sigma^{+} \leftrightarrow \mathrm{X}^{2} \Sigma^{+}$transition. (b) The FC factors for the $\mathrm{B}^{2} \Sigma^{+} \leftarrow \mathrm{X}^{2} \Sigma^{+}$ transition for $v=0,2$ and 14 for all upper vibrational levels up to $v=64$. Note the logarithmic scale used in both plots. 
spontaneous emission from $\mathrm{X}^{2} \Sigma^{+} v=64$ into the energetically accessible vibrational levels of the $\mathrm{A}^{2} \Pi$ state as just

$$
\tau^{-1}=\sum_{v=0}^{13} A_{64 v}=0.003 \mathrm{~s}^{-1}
$$

Furthermore, the individual $A_{64 v}$ coefficients lie in the narrow range $10^{-7}-10^{-5} \mathrm{~s}^{-1}$, despite the large changes in wavefunction phase suggesting strongly that the higher Feshbach levels will have similar spontaneous loss rates. This rate of decay is negligible so that the only decay modes available are fragmentation, collisional deactivation or IR emission within the $\mathrm{X}^{2} \Sigma^{+}$vibrational manifold. The above result also demonstrates that it is impractical to directly populate the $\mathrm{X}^{2} \Sigma^{+}$ Feshbach levels via the $\mathrm{A}^{2} \Pi$ state and higher electronic states must be used.

The $\mathrm{B}^{2} \Sigma^{+} a b$ initio points are fitted to a double well potential with the lowest vibrational level corresponding to a bound state in the inner well. Above $v=1$ vibrational levels corresponding to the outer potential well appear and our calculated $v=2$ level is consequently the lowest level of this potential minimum. The different states can be identified by the change in the rotational constant (from $\approx 1.5 \mathrm{~cm}^{-1}$ to $\approx 0.38 \mathrm{~cm}^{-1}$ ). The $\mathrm{B}^{2} \Sigma^{+}$and $\mathrm{C}^{2} \Sigma^{+}$states were calculated with an active orbital space including two additional non-valence $\mathrm{a}_{1}$ orbitals (8220) because of the presence of Rydberg excitations. Ab initio quantum chemistry is less suitable for the Rydberg potentials, although we have expanded the active space to calculate the lowest energy examples. The accuracy achieved is not as strong as for the valence states as can be clearly seen in Table 1 comparing the calculated potentials for the $\mathrm{B}^{2} \Sigma^{+}$(valence) and $\mathrm{C}^{2} \Sigma^{+}$(Rydberg) states with the experimental data. The present calculations could be improved by using additional diffuse orbitals in the atomic basis sets. Nevertheless, the calculations of the excited $\mathrm{C}^{2} \Sigma^{+}$state are both consistent with the experimental results of Novikov and Gurvich $^{15}$ and with $\mathrm{X}^{2} \Sigma^{+}$and $\mathrm{C}^{2} \Sigma^{+}$states belonging to the ${ }^{\prime} \mathrm{s}^{2} \Sigma^{+}$series of Rydberg states. Consequently, the $\mathrm{B}^{2} \Sigma^{+}$state is the lowest member of the ' $\mathrm{p}^{2} \Sigma^{+}$series. $^{45}$

As demonstrated in Fig. 4b, the FC factors from the ground state are negligible to the lowest vibrational states of this outer well. The sharp peaks in the figure correspond to vibronic transitions between the $\mathrm{X}^{2} \Sigma^{+}$state and the inner $\mathrm{B}^{2} \Sigma^{+}$ potential. Clearly for efficient population transfer a large FC factor is desirable and unfortunately excitation from any of the vibrational states populated through the cooling cycle is very small. However, a six-order of magnitude improvement is achieved by starting from the $v=8$ vibrational level (FC factor $\approx 0.002$ ) or higher. To transfer vibrational population between vibrational levels in the $\mathrm{X}^{2} \Sigma^{+}$state a pair of STIRAP pulses are used, though in the illustrated scheme (Fig. 3b) the higher $\mathrm{X}^{2} \Sigma^{+} v=14$ level is populated via the excited $\mathrm{D}^{2} \Pi$ $v=9$ level. To populate a level close to the lowest asymptote, however, we find that the $A$ coefficients reach a maximum (Fig. 4a) around $\mathrm{B}^{2} \Sigma^{+} v=45 \rightarrow \mathrm{X}^{2} \Sigma^{+} v=64$ despite the fact that the FC factors improve all the way to the highest levels of the $\mathrm{B}^{2} \Sigma^{+}$state $\left(0.1\right.$ for $\left.\mathrm{B}^{2} \Sigma^{+} v=64 \rightarrow \mathrm{X}^{2} \Sigma^{+} v=64\right)$. This reflects the fact that the transition dipole moment falls rapidly with internuclear distance as the atomic transition correlated to these molecular states is spin forbidden $\left(\operatorname{Be}\left({ }^{3} \mathrm{P}\right) \rightarrow \operatorname{Be}\left({ }^{1} \mathrm{~S}\right)\right.$ with an estimated decay rate ${ }^{58}$ of just $\left.0.4 \mathrm{~s}^{-1}\right)$. This peak rate is a fairly modest $9.2 \times 10^{2} \mathrm{~s}^{-1}$. The A coefficients starting from levels in the $\mathrm{D}^{2} \Pi$ state are even smaller, but this time the problem is the poor FC factors, not the transition dipole. We can understand this if we consider that the ion-pair potentials that form the minima of both the $\mathrm{X}^{2} \Sigma^{+}$and $\mathrm{A}^{2} \Pi$ states are very similar, thus the vibrational quanta in each state must be similar to maximize the wavefunction overlap.

It should be remembered, however, that it is not the $A$ coefficient that is directly relevant but the $B$ coefficient as STIRAP involves a stimulated, not spontaneous, emission step. The two emission rates are naturally related, though the $B$ coefficient can be defined in a number of slightly different forms. As experimentalists measure irradiance per unit area (typically $\mathrm{cm}^{-2}$ ) rather than unit volume we adopt a modified version of the definition popularised by Herzberg

$$
B_{v^{\prime} v^{\prime \prime}}^{\mathrm{GHz}}=\frac{10^{-3}}{2 \pi} B_{v^{\prime} v^{\prime \prime}}^{\omega} \mathbf{J}^{-1} \mathrm{~cm}^{2}(\mathrm{GHz})
$$

This definition should be more applicable for gas-phase absorption features and pulsed lasers (Table 2). The intensity of the STIRAP pulses is consequently another degree of control over the population transfer. The low transition rate in the final $\mathrm{B}^{2} \Sigma^{+} \rightarrow \mathrm{X}^{2} \Sigma^{+}$emission step can be ameliorated by using higher laser powers, an undemanding requirement as the transition frequency required lies in the visible region (see Section 4).

\begin{tabular}{|c|c|c|c|c|}
\hline & $\begin{array}{l}\text { Energy } \\
\left(\mathrm{cm}^{-1}\right) \\
{[\lambda(\mathrm{nm})]}\end{array}$ & $\begin{array}{l}A \text { coeffi- } \\
\text { cient } \\
\left(\mathrm{s}^{-1}\right)\end{array}$ & $\begin{array}{l}\mathrm{FC} \\
\text { factor }\end{array}$ & $\begin{array}{l}B \text { coefficient } \\
\left(\mathbf{J}^{-1}(\mathrm{GHz}) \mathrm{cm}^{2}\right)\end{array}$ \\
\hline \multicolumn{5}{|l|}{ Scheme 1} \\
\hline $\mathrm{D}^{2} \Pi \leftarrow \mathrm{X}^{2} \Sigma^{+}$ & 57299 & $1.8 \times 10^{6}$ & $3.2 \times 10^{-2}$ & $5.9 \times 10^{14}$ \\
\hline $9 \leftarrow 2$ & [174.6] & & & \\
\hline $\mathrm{D}^{2} \Pi \rightarrow \mathrm{X}^{2} \Sigma^{+}$ & 46091 & $9.7 \times 10^{5}$ & $1.5 \times 10^{-2}$ & $9.2 \times 10^{14}$ \\
\hline${ }_{\mathrm{D}^{2} \vec{\Sigma}^{+}}{ }^{4} \mathrm{Y}^{2} \boldsymbol{\Sigma}^{+}$ & {$[217.0]$} & & & \\
\hline $\begin{array}{l}\mathrm{B}^{2} \Sigma^{+} \leftarrow \mathrm{X}^{2} \Sigma^{+} \\
45 \leftarrow 14\end{array}$ & $\begin{array}{l}48590 \\
{[205.8]}\end{array}$ & $1.3 \times 10^{6}$ & $1.8 \times 10^{-3}$ & $7.5 \times 10^{14}$ \\
\hline $\begin{array}{l}\mathrm{B}^{2} \Sigma^{+} \rightarrow \mathrm{X}^{2} \Sigma^{+} \\
45 \rightarrow 64\end{array}$ & $\begin{array}{l}17013 \\
{[587.9]}\end{array}$ & $9.2 \times 10^{2}$ & $1.4 \times 10^{-3}$ & $6.8 \times 10^{12}$ \\
\hline \multicolumn{5}{|l|}{ Scheme 2} \\
\hline $\begin{array}{l}4^{2} \Pi \leftarrow X^{2} \Sigma^{+} \\
27 \leftarrow 2\end{array}$ & $\begin{array}{l}86370 \\
{[115.8]}\end{array}$ & $5.3 \times 10^{6}$ & $2.8 \times 10^{-2}$ & $5.0 \times 10^{14}$ \\
\hline $\begin{array}{l}4^{2} \Pi \rightarrow X^{2} \Sigma^{+} \\
27 \rightarrow 64\end{array}$ & $\begin{array}{l}41552 \\
{[240.7]}\end{array}$ & $6.3 \times 10^{5}$ & $2.6 \times 10^{-3}$ & $5.3 \times 10^{14}$ \\
\hline \multicolumn{5}{|l|}{ Scheme 3} \\
\hline $\begin{array}{l}\mathrm{A}^{2} \Pi \leftarrow \mathrm{X}^{2} \Sigma^{+} \\
3 \leftarrow 2\end{array}$ & $\begin{array}{l}34506 \\
{[289.8]}\end{array}$ & $7 \times 10^{6}$ & $1.9 \times 10^{-1}$ & $1.0 \times 10^{16}$ \\
\hline $\begin{array}{l}\mathrm{D}^{2} \Pi \leftarrow \mathrm{A}^{2} \Pi \\
6 \leftarrow 3\end{array}$ & $\begin{array}{l}21110 \\
{[473.7]}\end{array}$ & $1.1 \times 10^{6}$ & $3.0 \times 10^{-2}$ & $7.0 \times 10^{15}$ \\
\hline $\mathrm{D}^{2} \Pi \rightarrow \mathrm{X}^{2} \Sigma^{+}$ & 46670 & $8.1 \times 10^{6}$ & $5.1 \times 10^{-2}$ & $4.8 \times 10^{15}$ \\
\hline $6 \rightarrow 10 \mathrm{Y}^{2} \Sigma^{+}$ & [214.0] & & & \\
\hline $\begin{array}{l}\mathrm{A}^{2} \Pi \leftarrow \mathrm{X}^{2} \Sigma^{+} \\
10 \leftarrow 10\end{array}$ & $\begin{array}{l}32837 \\
{[304.5]}\end{array}$ & $2.4 \times 10^{6}$ & $4 \times 10^{-4}$ & $4.1 \times 10^{15}$ \\
\hline $\begin{array}{l}\mathrm{B}^{2} \Sigma^{+} \leftarrow \mathrm{A}^{2} \Pi \\
45 \leftarrow 10\end{array}$ & $\begin{array}{l}20278 \\
{[493.1]}\end{array}$ & $5 \times 10^{5}$ & $7.0 \times 10^{-2}$ & $3.6 \times 10^{15}$ \\
\hline $\begin{array}{l}\mathrm{B}^{2} \Sigma^{+} \rightarrow \mathrm{X}^{2} \Sigma^{+} \\
45 \rightarrow 64\end{array}$ & $\begin{array}{l}17013 \\
{[587.9]}\end{array}$ & $9.2 \times 10^{2}$ & $1.4 \times 10^{-3}$ & $6.8 \times 10^{12}$ \\
\hline
\end{tabular}

Table 2 Details of the STIRAP dissociation schemes proposed 


\section{(c) STIRAP population transfer via $4^{2} \Pi$ state}

An alternative STIRAP scheme involving the $4^{2} \Pi$ state was also explored to circumvent the relatively weak transition dipole from the vibrational levels close to the ${ }^{3} \mathrm{P}$ asymptote. To compute this state, a calculation of the lowest four ${ }^{2} \Pi$ states was conducted using a 7320 active space. At short range the second ${ }^{2} \Pi$ state is of Rydberg character, while at long range the 4th state is ion-pair in nature. The Rydberg component should have a strong absorption from the ground state but both the unconfirmed ${ }^{2} \Pi$ states observed by Novikov and Gurvich $^{15}$ lie at a rather higher energy. In Fig. 1 and Table 1 we report this $3^{2} \Pi$ Rydberg potential as a bound state, analogous to the $\mathrm{C}^{2} \Pi$ state in $\mathrm{CaF}$, crossed by the inner wall of the lower ion-pair ${ }^{2} \Pi$ state. However, the lack of experimental evidence suggests that such a Rydberg potential may be very heavily predissociated, so in the rest of the paper it is treated as forming the inner part of the $\mathrm{D}^{2} \Pi$ state.

The $4^{2} \Pi$ state correlates to the $\operatorname{Be}\left({ }^{1} \mathrm{P}\right)$ atom product and therefore has a strong electric dipole transition with the ground state even at extended bond lengths. Transitions into $\mathrm{X}^{2} \Sigma^{+} v=64$ are strong and increase above $v=45$ but as this transition is already very energetic (118 nm from $\left.\mathrm{X}^{2} \Sigma^{+} v=2\right)$ we use this state as a compromise (Fig. 5a). However a major difficulty arises from the high density of Rydberg states crossing this potential that will significantly increase the background coupling when compared to the $\mathrm{B}^{2} \Sigma^{+}$state. In Fig. 3 and 5 we have added a pair of schematic Rydberg potentials, based on our calculation for the lowest Rydberg, corresponding to those observed, exclusively to date, by Novikov and Gurvich. It should be noted that a vibrational progression was reported for both states so it appears that the predissociation suffered by these two states was not severe below the $\operatorname{Be}\left({ }^{3} \mathrm{P}\right)$ asymptote. Of more concern is the appearance of the ground potential of the $\mathrm{BeF}^{+}$ion well below the $4^{2} \Pi$ state. We have modelled this using our Rydberg potential, the ionization energy of the Be atom and the dissociation energy $(5.84 \mathrm{eV})$ calculated $^{27}$ by Ornellas et al. Its presence will strongly reduce the efficiency of the STIRAP process and so the lower energy $\mathrm{B}^{2} \Sigma^{+}$state is preferred for population transfer to the asymptote of the $\mathrm{X}^{2} \Sigma^{+}$potential.

\section{Discussion}

\section{(a) Technological requirements}

The STIRAP fragmentation of ultracold $\mathrm{BeF}$ replaces the need to produce a high power, continuous wave laser source in the XUV with laser cooling at around $305 \mathrm{~nm}$. Although the favoured STIRAP schemes involve the use of VUV light sources, the need for thousands of such photons for direct Doppler cooling has been replaced by, at most, a pair of laser pulses. In addition, even the shortest wavelength required in the STIRAP scheme here is significantly lower in energy than the F XUV cooling wavelength. Finally, while there is no known technology for the efficient production of a cw laser source in the XUV, the production of nanosecond pulses is possible in this wavelength region. Indeed, highly coherent, nanosecond XUV pulsed lasers have been developed for spectroscopy of atomic species such as $\mathrm{He}^{46}$ and molecules
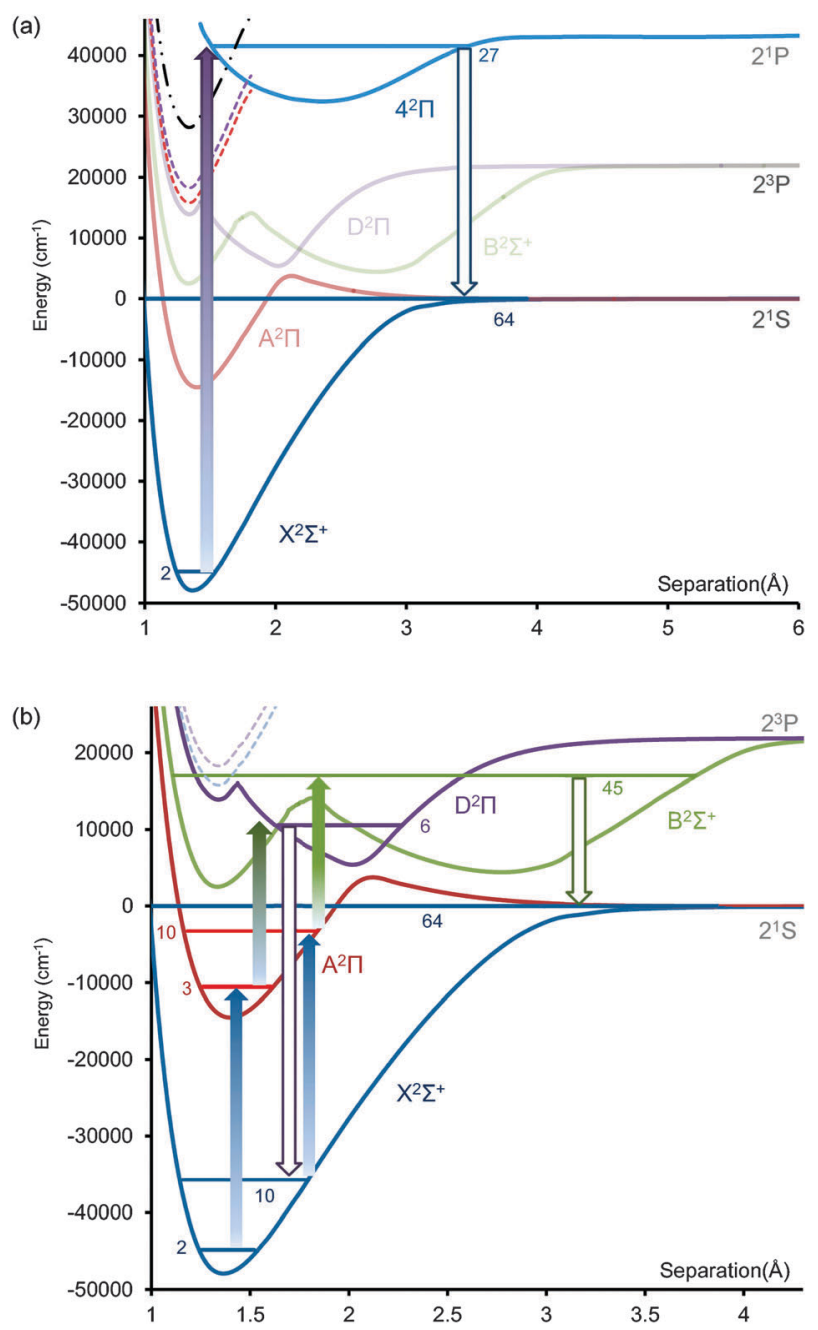

Fig. 5 Alternative STIRAP pathways to produce cold fluorine atoms (the final destination state is a Feshbach state formed within the hyperfine structure of the lowest spin-orbit asymptote for the interaction of ${ }^{9} \mathrm{Be}\left({ }^{1} \mathrm{~S}_{0}\right)$ and ${ }^{19} \mathrm{~F}\left({ }^{2} \mathrm{P}_{3 / 2}\right)$ atoms $)$. In the Hund's case (c) limit, the lowest state is $J=3 / 2$. The nuclear spin on the ${ }^{9} \mathrm{Be}$ atom is $3 / 2$ and $1 / 2$ on ${ }^{19} \mathrm{~F}$ producing hyperfine states $F=7 / 2,5 / 2,3 / 2$ and $1 / 2$. (a) Two pulse scheme with short wavelength $(118 \mathrm{~nm})$ pulse through the $4^{2} \Pi$ state; (b) $2 \times 3$-pulse scheme through $\mathrm{D}^{2} \Pi$ and $\mathrm{B}^{2} \Sigma^{+}$states, using $\mathrm{A}^{2} \Pi$ vibrational states as intermediate levels. The first group of pulses transfer population from $\mathrm{X}^{2} \Sigma^{+} v^{\prime \prime}=2$ to $v^{\prime \prime}=10$.

such as $\mathrm{CO}^{47}$ and $\mathrm{N}_{2}{ }^{48}$ and operate at sub $100 \mathrm{~nm}$ wavelengths. These sources are based on sum-frequency generation in an intense beam of gas $^{49}$ such as Xe, but the resulting pulse intensities are very low $\left(0.01 \mu \mathrm{J}\right.$ pulse $\left.{ }^{-1}\right)$. A tuneable VUV source operating over the range 110-170 $\mathrm{nm}$ has been developed that uses difference frequency generation in a similar arrangement $t^{50}$ and is capable of significantly higher pulse energies approaching $1 \mu \mathrm{J}$ pulse $^{-1}$.

For efficient STIRAP ultrafast pulses are usually ineffective but pulses of nanosecond duration have been used to demonstrate STIRAP in $\mathrm{Ne}^{51}$ atoms and $\mathrm{NO}^{52}$ molecules. However, in order to achieve the adiabaticity condition required for complete population transfer, shorter pulses need a higher intensity (in short, a high spectral intensity is necessary). Fortunately, in STIRAP the low power of the excitation pulse 
(PUMP) can be compensated using a more intense de-excitation (Stokes) pulse as the adiabaticity condition to be fulfilled is

$$
\sqrt{\Omega_{\mathrm{P}}^{2}+\Omega_{\mathrm{D}}^{2}} \geq \frac{1}{T}
$$

where $T$ is the duration of the pulses and $\Omega_{\mathrm{P}}$ and $\Omega_{\mathrm{D}}$ are Rabi frequencies. Note that in STIRAP the Stokes pulse is actually first and as the required wavelengths lie in the near UV or visible part of the spectrum, pulses with energy $0.1 \mathrm{~J}$ and above can be achieved. Still, it is clear that the transfer scheme in Fig. 3b would be at the very extreme of current technology.

Thus far we have specifically considered simple STIRAP schemes that involve either one or two pairs of pulses, but schemes involving additional intermediate levels have been discussed in the literature and these expand greatly the number of possible excitation pathways. Coulston and Bergmann ${ }^{53}$ investigated four- and five-branch systems and still achieved high population transfer efficiency. One particularly attractive scheme is so-called "straddle STIRAP" proposed by Tannor and co-workers ${ }^{54}$ which uses a number of intermediate states instead on just one. This would replace the short wavelength $\mathrm{D}^{2} \Pi \leftarrow \mathrm{X}^{2} \Sigma^{+}$excitation step in Fig. 3b with a two photon chain, $\mathrm{D}^{2} \Pi \leftarrow \mathrm{A}^{2} \Pi \leftarrow \mathrm{X}^{2} \Sigma^{+}$, replacing the VUV pulse with 300-500 nm pulses instead (Scheme 3, Table 2 and Fig. 5b). Now it is the turn of the intermediate $D^{2} \Pi \leftarrow A^{2} \Pi$ pulse to be the most intense but this is clearly easy to achieve with the wavelengths necessary. The minimization of emission loss from the first intermediate $\mathrm{A}^{2} \Pi$ level is important, but due to the strongly diagonal FC factors any population decay will feed back into the laser cooling cycling. The final excitation step can also be replaced with an equivalent $\mathrm{B}^{2} \Sigma^{+} \leftarrow \mathrm{A}^{2} \Pi \leftarrow \mathrm{X}^{2} \Sigma^{+}$ sequence. This would relax the problem of fulfilling the adiabaticity condition above with VUV pulses.

Since the key requirement of the first pulse sequence is to simply populate a high vibrational level of the $\mathrm{X}^{2} \Sigma^{+}$state, such as $v^{\prime \prime}=10$, an alternative method would be to exploit the growing strength of $\Delta v=1$ and $\Delta v=2$ vibrational changes in the $\mathrm{A}^{2} \Pi \leftarrow \mathrm{X}^{2} \Sigma^{+}$transition (Fig. 2a) to populate this vibronic state via the chain $\mathrm{X}^{2} \Sigma^{+} v^{\prime \prime}=10 \leftarrow v^{\prime \prime}=6 \leftarrow v^{\prime \prime}=4 \leftarrow v^{\prime \prime}=2$. All the required excitation steps would lie in the UV/visible region.

\section{(b) Further issues}

The effect of background electronic states on the population transfer is a crucial issue because of the presence of Rydberg states below the excited dissociation limits studied here. Despite the presence of these Rydberg states in the vicinity of the $\mathrm{B}^{2} \Sigma^{+}$state, numerous theoretical studies have shown that predissociation of the excited state is not a serious issue because in the STIRAP process the excited state is never actually populated. Kurkal and Rice ${ }^{55}$ have demonstrated that even for polyatomic molecules population transfer in the presence of off-resonance radiative coupling is insensitive to these background states. Furthermore, it is also possible to transfer into a predissociating state with unity efficiency, even if the process is not strictly adiabatic. ${ }^{56}$

The exception to this general robustness is the presence of background ionization processes which would be unavoidable for excitation through the $4^{2} \Pi$ state. Furthermore, an infinite series of Rydberg states will cross the $4^{2} \Pi$ state at the inner wall as $4^{2} \Pi$ lies above the $\mathrm{X}^{1} \Sigma^{+}$ground state of the $\mathrm{BeF}^{+}$ion. Indeed, any electronic state above the 1 st excited $2^{3} \mathrm{P}$ asymptote could suffer from extremely high decay rates. The presence of these Rydbergs also implies there may only be a small window of $\mathrm{B}^{2} \Sigma^{+}$vibrational levels suitable for complete population transfer. Note that the lowest vibrational levels of the two alleged ${ }^{2} \Pi$ Rydberg states ${ }^{15}$ lie above $\mathrm{B}^{2} \Sigma^{+} v=45$. A lower $\mathrm{B}^{2} \Sigma^{+}$vibrational level will suffer less overlap with Rydberg states but at a cost with respect to the final Stokes transition dipole (for example, the $v=41$ level lies $1990 \mathrm{~cm}^{-1}$ below $v=45$ but the corresponding $A$-coefficient is a third of the magnitude).

It is therefore worth estimating how many Rydberg states may exist below the $\mathrm{B}^{2} \Sigma^{+} v=45$ level. The highest Rydberg states we have calculated correspond to the lowest $n=3$ states of ${ }^{2} \Sigma^{+}$and ${ }^{2} \Pi$ symmetry and lie at lower energy. Such low lying Rydberg states tend to still have a strong valence component. However, the next shell states are more regular in nature which we can demonstrate by calculating the following

$$
E_{\mathrm{R}}=\frac{E_{\infty}-E_{n+2}}{Z^{2}}
$$

for a series of one electron systems such as the alkali atoms and the alkaline earth ions (Table 3 ). Here $Z$ is the charge on the ionized state, $E_{\infty}$ is the ionization energy, $E_{n+2}$ is the energy of $(n+2)$ p state and $n$ is the valence shell quantum number. Despite the considerable differences in energies of the individual levels and with the exception $\mathrm{H}(n=1)$, the calculated energy term $E_{\mathrm{R}}$ lies within a relatively compact energy range, though it falls steadily with increasing valence $n$ as expected. This can then be compared with an equivalent molecular Rydberg state in CaF. Here

$$
n^{*}=n-\mu
$$

where $n^{*}$ is the reduced principal quantum number. We have chosen $\mathrm{p}$ states for all species because the quantum defects $\mu$ of the ${ }^{\prime}{ }^{2} \Sigma^{+}$states are among the smallest in CaF. ${ }^{45}$ The resulting value for $E_{\mathrm{R}}$ is much smaller than the equivalent value for $\mathrm{Ca}^{+}$, a result of the additional charge-dipole interaction term in the molecular potentials. Consequently this Rydberg lies closer to the ionized state and strongly suggests that the corresponding $E_{\mathrm{R}}$ for $\mathrm{Be}^{+}$is also an upper limit for

Table 3 Details of the ionization potentials and Rydberg energies of one electron systems, including $\mathrm{CaF}$ (see text for further details)

\begin{tabular}{lcrrr}
\hline Element & $n$ & \multicolumn{1}{c}{$E_{\infty}$} & \multicolumn{1}{c}{$E_{n+2}$} & \multicolumn{1}{c}{$E_{\mathrm{R}}$} \\
\hline $\mathrm{H}$ & 1 & 109678 & 97492 & 12186 \\
$\mathrm{Li}$ & 2 & 43487 & 36469 & 7018 \\
$\mathrm{Be}^{+}$ & 2 & 146882 & 118760 & 7030 \\
$\mathrm{Na}$ & 3 & 41449 & 35040 & 6409 \\
$\mathrm{Mg}^{+}$ & 3 & 121267 & 97455 & 5953 \\
$\mathrm{~K}$ & 4 & 35009 & 28999 & 6010 \\
$\mathrm{Ca}^{+}$ & 4 & 95751 & 74484 & 5316 \\
$\mathrm{Rb}^{+}$ & 5 & 33690 & 27835 & 5855 \\
$\mathrm{Sr}^{+}$ & 5 & 88964 & 68645 & 5079 \\
$\mathrm{Cs}$ & 6 & 31406 & 25708 & 5698 \\
$\mathrm{Ba}^{+}$ & 6 & 80686 & 61339 & 4836 \\
$\mathrm{CaF}$ & $(4)$ & 46998 & $43826^{a}$ & 3172 \\
${ }^{a}$ Energy of $5.88{ }^{\circ} \mathrm{p}^{2} \Sigma^{+}$state. & & \\
\hline
\end{tabular}


the equivalent Rydberg state of the molecular species BeF. Again using the $\mathrm{BeF}^{+}$dissociation energy calculated ${ }^{27}$ by Ornellas et al., our calculated dissociation energy for $\mathrm{BeF}$ $\mathrm{X}^{2} \Sigma^{+}$, the ionization energy of $\mathrm{Be}$ and the value of $E_{\mathrm{R}}$ for $\mathrm{Be}^{+}$, we are confident that no Rydberg states higher than $n^{*}=3$ exist below $\sim 68700 \mathrm{~cm}^{-1}$, more than $5000 \mathrm{~cm}^{-1}$ above $\mathrm{B}^{2} \Sigma^{+} v=45$. Consequently, there are a maximum of five missing Rydberg states in Fig. 1 that may predissociate this vibrational level, which should not severely interfere with the STIRAP process.

A further advantage to the proposed scheme involves the elimination of photon recoil heating by the STIRAP sequence. There is no recoil heating effect from STIRAP because there is no spontaneous emission and therefore no dissipation. A momentum kick is inevitable, however, because there is (usually) a mismatch between the frequencies of the pump and dump pulses. This net momentum kick, however, is the same for all the parent molecules and serves to impart an impulse to the centre-of-mass and can be nullified by applying an external field and exploiting the paramagnetic nature of the fluorine atom.

\section{Conclusion}

We have calculated the low lying electronic states of $\mathrm{BeF}$ in order to investigate the possibility of laser cooling the molecule and then using optical pulses to effect a zero-energy fragmentation of the parent. A four colour laser scheme would appear to be effective for cooling. A STIRAP excitation process via the $\mathrm{B}^{2} \Sigma^{+}$state appears feasible though the transition dipole is small. Higher states are complicated by the presence of short range Rydberg states and the appearance of the ground state of the molecular ion well below the second excited asymptote of the neutral. Although STIRAP is an efficient technique, the production of ultracold parent molecules requires dense clouds of Doppler cooled diatomics and the multiple cooling wavelengths that entails. Crucially, however, the cold atoms formed can be elements that are difficult or impossible to cool with current technology. Thus, it is not a competitor to traditional laser cooling techniques but a technology to be adopted when these methods are inadequate.

A more general observation concerns the need to fulfill the dual requirements of a parent diatomic that can be laser cooled and a molecule that can be subsequently fragmented at the dissociation limit via STIRAP. The best laser cooling candidates identified so far $^{57}$ are either ionic compounds or hydrides and the former are characterised by very deep ground state potentials. Consequently, large energy separations need to be bridged, requiring either short wavelength photons or a chain of intermediate levels to break these molecules apart. However, the problem of absorbing tens of thousands of XUV photons (setting aside for one moment the lack of such laser sources in the foreseeable future) to cool a halogen has been reduced to using conventional $\mathrm{cw}$ lasers in concert with a series of excitation pulses in the UV and visible. The final temperature will not be limited by the concomitant large recoil heating that would be unavoidable in direct Doppler cooling of fluorine.

The lack of a zero spin, stable isotope of Be complicates the cooling sequence because of the increased number of hyperfine levels that need to be pumped within each vibronic transition. This problem must be addressed in the cooling of other fluorides, such as ${ }^{137} \mathrm{BaF}$. In addition, BeF possesses the largest dissociation energy of the alkaline earth fluorides. Alternative Group 2 fluorides with zero spin metal atoms, such as $\mathrm{MgF}$, $\mathrm{CaF}$ or $\mathrm{SrF}$, with simple hyperfine structures and weaker ionic bonds, may prove to be more practical fluorine sources.

While our calculations suggest grounds for cautious optimism, a number of important issues remain to be explored. A theoretical or spectroscopic determination of the quantum defects and a subsequent Quantum Defect Theory (QDT) analysis of the BeF Rydberg states would help clarify the feasibility of the proposed excitation scheme. A calculation of the long-range Feshbach states based on the atomic polarizabilities will refine the FC factors and $A$-coefficients estimated here. Finally, a simulation of the multi-pulse sequences proposed with realistic pulse energies and durations is desirable to determine under what precise conditions the populations of the penultimate level is zero and the first intermediate level is minimised.

\section{Acknowledgements}

The author would like to thank Adam Turner and Daniel Rogers for their help with the present calculations.

\section{References}

1 K.-K. Ni, S. Ospelkaus, D. Wang, G. Quemener, B. Neyenhuis, M. H. G. de Miranda, J. L. Bohn, J. Ye and D. S. Jin, Nature, 2010, 464, 1324-1328.

2 W. S. Struve, T. Kitagawa and D. R. Herschbach, J. Chem. Phys., 1971, 54, 2759-2761.

3 C. Liu and J. Noonan, Advanced Photon Source Accelerator Ultrahigh Vacuum Guide, ANL/APS/TB-16, Advanced Photon Source, Argonne National Laboratory, USA.

4 K. W. A. Wright and I. C. Lane, Phys. Rev. A: At., Mol., Opt. Phys., 2010, 82, 032715 .

5 K. Lidén, Ark. Fys., 1949, 1, 229.

6 M. Shimizu, K. Yasutake, H. Ohmi, A. Takeuchi, H. Kakiuchi, K. Yoshii and Y. Mori, Appl. Phys. B: Lasers Opt., 2001, 72, 227-230.

7 E. S. Shuman, J. F. Barry and D. DeMille, Nature, 2010, 467, $820-823$.

8 E. S. Shuman, J. F. Barry, D. R. Glenn and D. DeMille, Phys. Rev. Lett., 2009, 103, 223001.

9 M. Pelegrini, C. S. Vivacqua, O. Roberto-Neto, F. R. Ornellas and F. B. C. Machado, Braz. J. Phys., 2005, 35, 950-956.

10 S. Datta, Proc. R. Soc. London, Ser. A, 1922, 101, 187.

11 F. A. Jenkins, Phys. Rev., 1930, 35, 315-335.

12 T. E. H. Walker and R. F. Barrow, J. Phys. B: At., Mol. Phys., 1969, 2, 102-106.

13 D. L. Cooper, S. J. Prosser and W. G. Richards, J. Phys. B: At. Mol. Phys., 1981, 14, L487-L489.

14 K. M. Rao and P. T. Rao, Indian J. Pure Appl. Phys., 1965, 3, 177.

15 M. M. Novikov and L. V. Gurvich, Opt. Spektrosk Engl. Transl., 1967, 23, 173.

16 G. Tai and R. D. Verma, J. Mol. Spectrosc., 1995, 173, 1-7.

17 A. C. Roach and P. J. Kuntz, Mol. Phys., 1982, 45, 853-856.

18 D. L. Hildenbrand and E. J. Murad, J. Chem. Phys., 1966, 44, $1524-1529$.

19 M. Farber and R. D. Srivastava, J. Chem. Soc., Faraday Trans. 1, 1974, 70, 1581-1589.

20 M. Vasiliu, D. Feller, J. L. Gole and D. A. Dixon, J. Phys. Chem. A, 2010, 114, 9349-9358.

21 T. E. H. Walker and W. G. Richards, J. Phys. B: At. Mol. Phys., 1970, 3, 271-279. 
22 T. E. H. Walker and W. G. Richards, Proc. Phys. Soc., 1967, 92, 285-290.

23 C. M. Marian, Chem. Phys., 1985, 100, 13-19.

24 S. R. Langhoff, C. W. Bauschlicher and H. Partridge, J. Chem. Phys., 1986, 84, 1687-1695.

25 S. R. Langhoff, C. W. Bauschlicher, H. Partridge and R. Ahlrichs, J. Chem. Phys., 1986, 84, 5025-5031.

26 F. B. C. Machado and F. R. Ornellas, Mol. Phys., 1989, 67, $1129-1140$.

27 F. R. Ornellas, F. B. C. Machado and O. Roberto-Neto, Mol. Phys., 1992, 77(6), 1169-1185.

28 Z. L. Zhu, Q. P. Song, S. H. Kou, J. H. Lang and J. Feng, Int. J. Mol. Sci., 2012, 13, 2501-2514.

29 S. J. Prosser and W. G. Richards, J. Phys. B: At. Mol. Phys., 1980, 13, 2767-2772.

30 H.-J. Werner and P. J. Knowles, J. Chem. Phys., 1985, 82, 5053-5063.

31 H.-J. Werner and P. J. Knowles, J. Chem. Phys., 1988, 89, 5803-5814.

32 S. R. Langhoff and E. R. Davidson, Int. J. Quantum Chem., 1974, 8, 61-72.

33 D. E. Woon, T. H. Dunning Jr, unpublished; D. Feller, J. Comput. Chem., 1996, 17, 1571-1586.

34 H.-J. Werner, P. J. Knowles, R. Lindh, F. R. Manby, M. Schütz, P. Celani, T. Korona, A. Mitrushenkov, G. Rauhut, T. B. Adler, R. D. Amos, A. Bernhardsson, A. Berning, D. L. Cooper, M. J. O. Deegan, A. J. Dobbyn, F. Eckert, E. Goll, C. Hampel, G. Hetzer, T. Hrenar, G. Knizia, C. Köppl, Y. Liu, A. W. Lloyd, R. A. Mata, A. J. May, S. J. McNicholas, W. Meyer, M. E. Mura, A. Nicklass, P. Palmieri, K. Pflüger, R. Pitzer, M. Reiher, U. Schumann, H. Stoll, A. J. Stone, R. Tarroni, T. Thorsteinsson, M. Wang and A. Wolf, "Molpro, version 2010.1, a package of ab initio programs", 2009.

35 R. J. Le Roy, LEVEL 8.0: A computer program for solving the radial Schrödinger equation for bound and quasibound Levels, Chemical Physics Research Report CP-663, University of Waterloo, 2007 the source code and manual for this program may be obtained from http://leroy.uwaterloo.ca/programs/.

36 T. C. Steimle, S. Frey, A. Le, D. DeMille, D. A. Rahmlow and C. Linton, Phys. Rev. A: At., Mol., Opt. Phys., 2011, 84, 012508.

37 L. B. Knight Jr., M. B. Wise, A. G. Childers, E. R. Davidson and W. R. Daasch, J. Chem. Phys., 1980, 73, 4198-4202.

38 D. Patterson, E. Tsikata and J. M. Doyle, Phys. Chem. Chem. Phys., 2010, 12, 9736-9741.

39 N. P. Wells and I. C. Lane, Phys. Chem. Chem. Phys., 2011, 13, 19036-19051.
40 H. E. Radford, V. W. Hughes and V. Beltran-Lopez, Phys. Rev., 1961, 123, 153-160.

41 N. V. Vitanov, T. Halfmann, B. W. Shore and K. Bergmann, Annu. Rev. Phys. Chem., 2001, 52, 763-809.

42 K. Winkler, F. Lang, G. Thalhammer, P. V. D. Straten and J. H. Denschlag, Phys. Rev. Lett., 2007, 98, 043201.

43 S. Ospelkaus, K.-K. Ni, H. G. de Miranda, B. Neyenhuis, D. Wang, S. Kotochigova, P. S. Julienne, D. S. Jin and J. Ye, Faraday Discuss., 2009, 142, 351-360.

44 J. G. Danzl, M. J. Mark, E. Haller, M. Gustavsson, R. Hart, J. Aldegunde, J. M. Hutson and H.-C. Nägerl, Nat. Phys., 2010, 6, 265-270.

45 J. J. Kay, S. L. Coy, B. M. Wong, C. Jungen and R. W. Field, J. Chem. Phys., 2011, 134, 114313.

46 K. S. E. Eikema, W. Ubachs, W. Vassen and W. Hogervorst, Phys. Rev. A: At., Mol., Opt. Phys., 1997, 55, 1866-1884.

47 F. P. Levelt, W. Ubachs and W. Hogervorst, J. Chem. Phys., 1992, 97, 7160-7166.

48 J. P. Sprengers, W. Ubachs, K. G. H. Baldwin, B. R. Lewis and W.-Ü L. Tchang-Brillet, J. Chem. Phys., 2003, 119, 3160-3173.

49 W. Ubachs, K. S. E. Eikema, W. Hogervorst and P. C. Cacciani, J. Opt. Soc. Am. B, 1997, 14, 2469-2476.

50 S. J. Hanna, P. Campuzano-Jost, E. A. Simpson, D. B. Robb, I. Burak, M. W. Blades, J. W. Hepburn and A. K. Bertram, Int. J. Mass Spectrom., 2009, 279, 134-146.

51 J. Martin, B. W. Shore and K. Bergmann, Phys. Rev. A: At., Mol., Opt. Phys., 1999, 54, 1556-1569.

52 A. Kuhn, S. Steuerwald and K. Bergmann, Eur. Phys. J. D, 1998, $1,57-70$

53 G. W. Coulston and K. Bergmann, J. Chem. Phys., 1992, 96, $3467-3475$.

54 V. S. Malinovsky and D. J. Tannor, Phys. Rev. A: At., Mol., Opt. Phys., 1997, 56, 4929-4937.

55 V. Kurkal and S. A. Rice, J. Phys. Chem. B, 2001, 105, 6488-6494.

56 V. Kurkal and S. A. Rice, J. Phys. Chem. A, 2002, 106 , 10810-10817.

57 M. D. Di Rosa, Eur. Phys. J. D, 2004, 31, 395-402.

58 As there is no experimental measurement available, this value is estimated from the calculated relative oscillator strengths of L. Głowacki and J. Migdałek, J. Phys. B: At. Mol. Opt. Phys., 2006, 39, 172 and the experimental measurement $(4.4 \mathrm{~ms})$ in Mg I from P. L. Hansen, K. T. Therkildsen, N. Malossi, B. B. Jensen, E. D. van Ooijen, A. Brusch, J. H. Müller, J. Hald and J. W. Thomsen, Phys. Rev. A: At., Mol., Opt. Phys., 2008, 77, 062502 . 